\title{
Characterization of a Cold Cesium Source for PARCS: Primary Atomic Reference Clock in Space
}

\author{
T. P. Heavner, L. Hollberg, S. R. Jefferts, J. Kitching, W. M. Klipstein, D. M. Meekhof, and H. G. Robinson
}

\begin{abstract}
The Primary Atomic Reference Clock in Space (PARCS) project is a joint NIST-JPL-University of Colorado venture aimed at placing a Cs atomic clock aboard the International Space Station (ISS). This orbiting clock will achieve high accuracy, in part due to the long Ramsey times afforded by the microgravity environment, and allow for precision tests of fundamental physics including relativity theory. As part of this effort, we are evaluating the characteristics of a prototype cold Cs source based on launching atoms from an optical molasses. Experimental results, in conjunction with theoretical modeling of atom flux requirements, will be applied to the design and construction of a robust, space-qualified device. The apparatus described here will be used to develop other PARCS components such as the microwave cavity structure and detection systems, and to investigate two-dimensional cooling schemes for future $\mathrm{Cs}$ fountains and space clocks.
\end{abstract}

\section{INTRODUCTION}

$\mathbf{L}$ ASER cooling techniques have allowed the development of modern fountain clocks that now achieve uncertainties of $\approx 1 \times 10^{-15},[1]$, [2]. The maximum practical Ramsey time achievable in a fountain is on the order of $1 \mathrm{~s}$. Recently, several groups have begun the development of space-based clocks in order to obtain the longer Ramsey times $(\sim 10 \mathrm{~s})$ possible in a microgravity environment, to perform tests of fundamental physics such as relativity theory, and to provide a globally available realization of the second.

Our group at the National Institute of Standards and Technology (NIST) is collaborating with Jet Propulsion Laboratory (JPL) and the University of Colorado to develop Primary Atomic Reference Clock in Space (PARCS), a Cs atomic clock that is scheduled to fly aboard the International Space Station (ISS). An overview of the PARCS space clock project has been presented elsewhere [3]. Similarly, the PHARAO [4] and RACE [5] space clock projects address many of the same issues and problems. Our present goal is to achieve a stability of $\sigma_{y}(\tau) \approx 5 \times 10^{-14} \tau^{-1 / 2}$ with an ultimate uncertainty of $\sim$ $10^{-16}$. The final clock parameters will depend on many factors, some of which are still under study. One of our roles in this collaboration has been to develop a laboratory prototype cold Cs source that, along with theoretical modeling of atom flux requirements, will provide design parameters to aid JPL in the construction of a robust, space qualified apparatus.

Manuscript received May 14, 2000; revised November 1, 2000.

T. P. Heavner, L. Hollberg, S. R. Jefferts, J. Kitching, D. M. Meekhof, and H. G. Robinson are with the Time and Frequency Division, National Institute of Standards and Technology, Boulder, CO 80303 USA.

W. M. Klipstein is with the Jet Propulsion Laboratory, California Institute of Technology, Pasadena, CA 91109 USA.

Publisher Item Identifier S 0018-9456(01)02599-2.
PARCS will operate in the configuration illustrated in Fig. 1. Cold Cs atoms are launched from an optical molasses [6], state selected, then enter a Ramsey-type microwave cavity. The shutters allow the clock to operate in a multiple launch mode; that is, a second ball of atoms can be loading in the source region while the previous ball is in the interrogation region, thus increasing the flux of atoms through the clock. Upon exiting the interrogation region, the atoms enter a detection region where the populations in $F=3$ and $F=4$ are measured.

We have developed theoretical models of atom flux requirements that yield a range of operating parameters which achieve $\sigma_{y}(\tau) \approx 5 \times 10^{-14} \tau^{-1 / 2}$. Consider a clock with Ramsey cavities separated by $75 \mathrm{~cm}$. With a molasses load time of $0.3 \mathrm{~s}$, an atom temperature of $2 \mu \mathrm{K}$, a Ramsey time of $5 \mathrm{~s}$, and 4 launches per lineside, we require $\approx 2 \times 10^{6}$ atoms in the $m_{F}=0$ clock state per launch. The same conditions with a $10 \mathrm{~s}$ Ramsey time boosts the required number of atoms to $5 \times 10^{6}$.

\section{EXPERIMENTAL APPARATUS}

Our experimental apparatus is shown in Fig. 2. The vacuum chamber consists of the source region, a long toss tube $(\sim 1.5$ $\mathrm{m}$ ), and a detection region. The source region contains twelve $3.8 \mathrm{~cm}$ windows. Six windows are located on the faces of a cube oriented in the $(1,1,1)$ beam geometry. These are used for introducing the cooling beams into the vacuum. The remaining six windows are for viewing and for future experimental studies. There are several smaller ports containing a Cs oven and a small window suitable for introducing a Zeeman slower beam. This system contains three vacuum pumps: an ion pump located at the top of the toss tube, graphite getters located near the center of the source, and a Ti sublimation pump between the source and the toss tube. The source region is surrounded by three pairs of Helmholtz coils for zeroing the ambient magnetic field.

An optical amplifier pumped by a distributed Bragg reflector (DBR) diode laser supplies $\sim 200 \mathrm{~mW}$ of light at $852 \mathrm{~nm}$. The laser light is passed through an optical isolator and then divided into two beams that are delivered to separate double-pass acoustic-optic modulators (AOM) allowing the three upward pointing beams to be shifted in frequency independently from the three downward beams for launching. Nominally, the beams are shifted by the AOMs and are detuned $\sim 2 \Gamma$ red of the $F=$ $4 \rightarrow F^{\prime}=5$ transition. The beams are then further split and approximately equal powers are coupled into six polarizationmaintaining fiber-optic cables. The fibers extend to output couplers mounted on the chamber windows that expand the beams to a $1 / e^{2}$ diameter of $\sim 1.5 \mathrm{~cm}$. The fiber output couplers contain polarizing beamsplitting cubes that direct the beam originating from the opposite output coupler onto a photodiode. This 


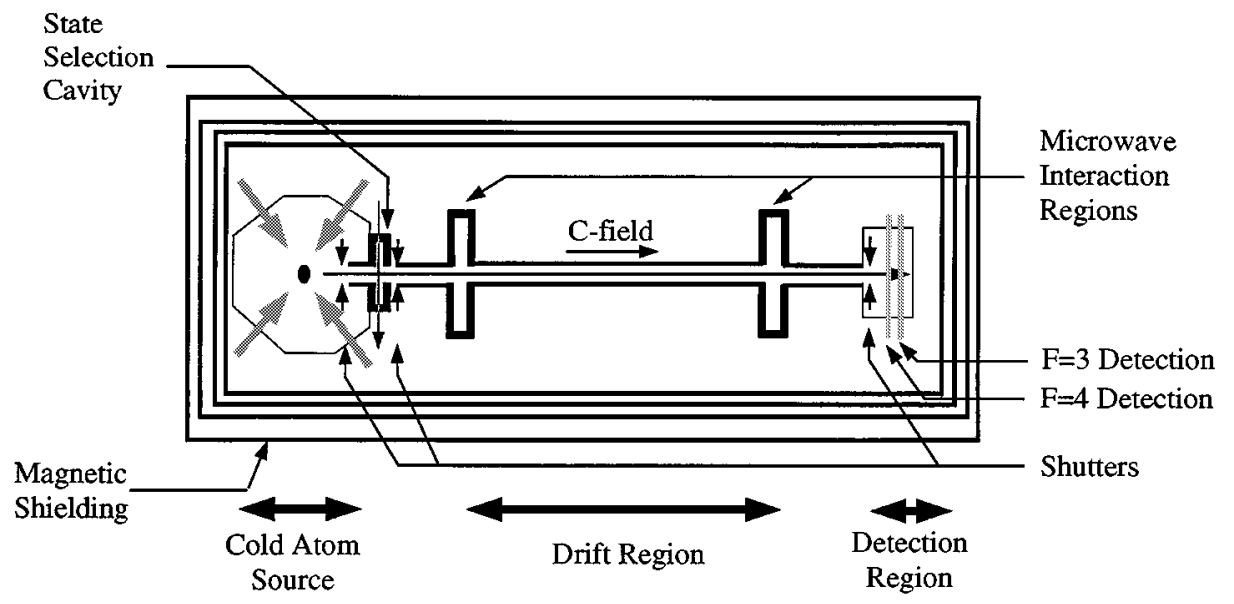

Fig. 1. Schematic diagram of the PARCS cesium clock physics package. The C-field region encompasses the source and the detection regions.

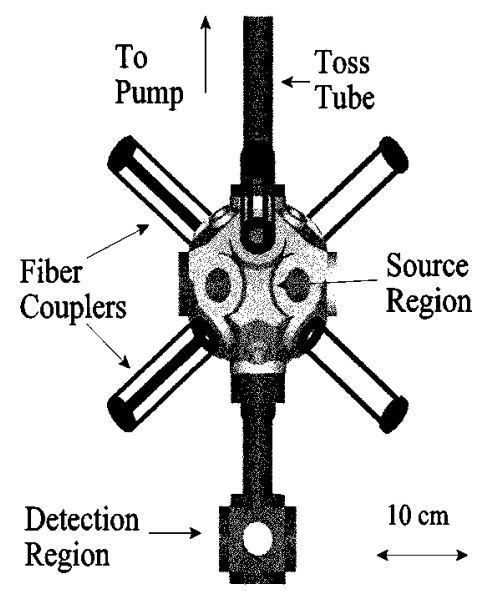

Fig. 2. Cold Cs source experimental apparatus.

allows the intensity of the six cooling beams to be monitored in real time. We have developed an intensity servo for the six beams; the servo monitors the light levels at these photodiodes and then sends control signals back onto variable retarders on the optical table which in turn adjust the amount of light entering the six fiber input couplers.

An extended-cavity diode laser (ECDL) locked on the $F=$ $3 \rightarrow F^{\prime}=4$ transition serves as a repump $(\sim 5 \mathrm{~mW})$ and this is directed onto the molasses region through one of the additional side windows. A beam from a second ECDL locked on the $F=4 \rightarrow F^{\prime}=5$ transition is delivered via a polarization-maintaining fiber to the vacuum system where cylindrical lenses expand the beam into a sheet of light $\sim 0.5 \mathrm{~mm}$ thick, after which it enters a window on the detection cube that is located $\sim 25 \mathrm{~cm}$ below the source. A retro mirror directs the detection beam back onto itself and creates a standing wave. The detection system consists of collection optics, a large photodiode and a high-gain amplifier and is located on one of the remaining windows of the detection cube. Calibration of the detection system allows the number of atoms in the ball to be determined. Additional information on atom number is provided by a charge-coupled device (CCD) imaging system focused onto the molasses region.

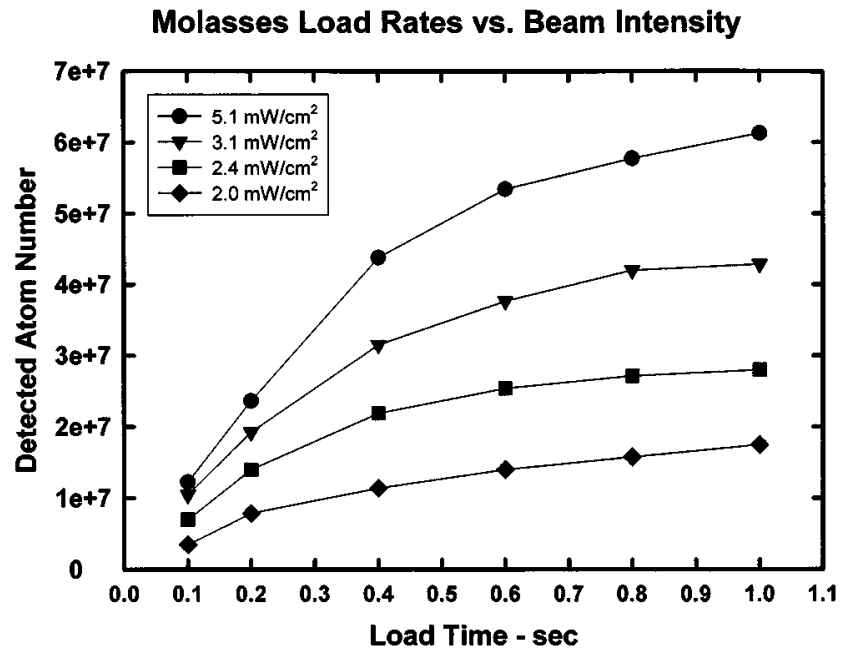

Fig. 3. Number of Cs atoms arriving at the detection region after dropping from the optical molasses at various load times and beam intensities.

We operate the apparatus in two basic modes: 1) dropping atoms and 2) launching atoms. In the drop mode, the molasses beams are turned on for a time $T_{\text {load }}$, the atoms are then subjected to a post-cool routine by detuning the beams to $\approx-8 \Gamma$ from the $F=4 \rightarrow F^{\prime}=5$ transition, and finally the beams are shut off allowing the atoms to fall to the detection region. In the launch mode, the molasses beams are on for $T_{\text {load }}$, the up beams are then detuned with respect to the down beams creating a moving molasses at the launch velocity. Approximately $2 \mathrm{~ms}$ later, the atoms are post-cooled before leaving the source region. The atoms travel up, reach apogee, and fall back into the detection region.

\section{EXPERIMENTAL RESULTS}

We have measured the molasses loading rate as a function of a variety of parameters. Fig. 3 shows data obtained by dropping atoms from the molasses after various load times and for different beam intensities at a constant beam diameter of $\sim 1.5 \mathrm{~cm}$ $\left(1 / e^{2}\right)$. For example, at a load time of $1 \mathrm{~s}$, with intensities of 


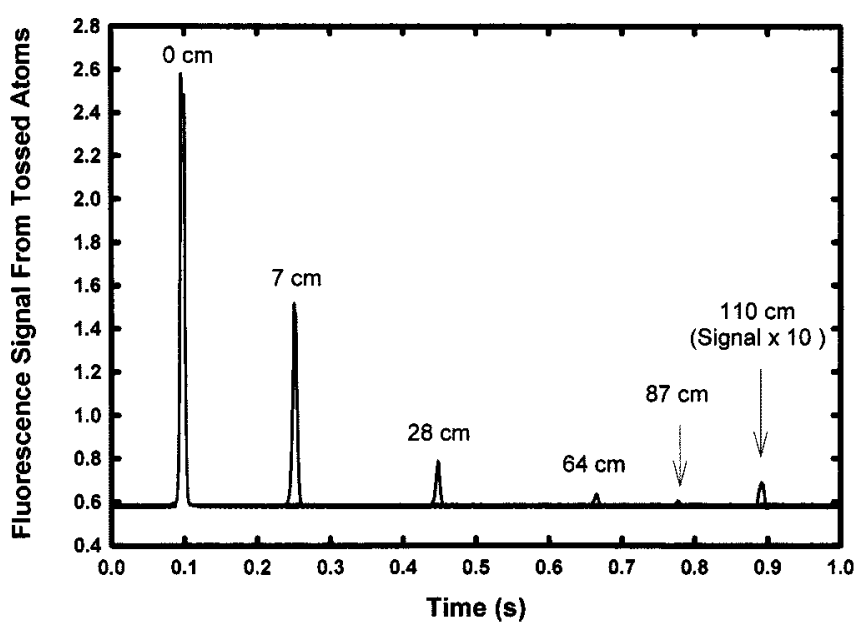

Fig. 4. Plot of the fluorescence signal of atoms in the detection region after tossing to various heights.

$\sim 5 \mathrm{~mW} / \mathrm{cm}^{2}$ per beam, $\sim 6 \times 10^{7}$ atoms arrive in the detection region. With the present experimental configuration, we are limited to intensities of $\sim 5 \mathrm{~mW} / \mathrm{cm}^{2}$.

By recording the size of the atomic fluorescence signal in the detection region as a function of various launch heights (shown in Fig. 4) we can determine the temperature of the atom sample. Such measurements indicate an atom temperature of $2.8 \mu \mathrm{K} \pm$ $0.8 \mu \mathrm{K}$ and an initial radius of $\sim 0.5 \mathrm{~cm}$. We anticipate that recent improvements in the launch and post-cool routines will produce still lower atom temperatures. Approximately $20 \%$ of the atoms in the molasses are lost during the drop at a $\sim 1.3 \mathrm{~cm}$ diameter aperture located between the source and the detection region. This loss factor applied to the results from the dropped signal in Fig. 3 with $5 \mathrm{~mW} / \mathrm{cm}^{2}$ per beam at long load times indicates that the number originally in the source is $\sim 8 \times 10^{7}$. This value is consistent with the number of atoms measured within the molasses from fluorescence using the CCD imaging system.

We can compare the results of these measurements with the requirements outlined by our flux modeling. For example, at a load time of $\sim 0.3 \mathrm{~s}$ (this is the load time our present flux models incorporate) with $5 \mathrm{~mW} / \mathrm{cm}^{2}$ per beam there are $\sim 5 \times 10^{7}$ atoms originally in the source. Assuming equal populations among the nine $m_{F}$ sub levels, this leaves $\sim 6 \times 10^{6}$ atoms in the $F=$ $4, m_{F}=0$ state. According to our flux requirements model, this allows for a $10 \mathrm{~s}$ Ramsey time and a stability of $\sigma_{y}(\tau) \approx$ $5 \times 10^{-14} \tau^{-1 / 2}$ (assuming a target atom temperature of $2 \mu \mathrm{K}$ ).

We have also characterized our Cs oven to determine how the atoms load into the molasses. In one experiment, we turned on the Cs oven and monitored the number of atoms in a dropped signal with a constant load time as the oven heated up. The oven quickly reached its set-point temperature, and during this initial warm-up the number of atoms in the signal increased at the same rate as the oven temperature. However, even after the temperature of the oven stabilized, the number of atoms in the signal continued to increase significantly for several hours. This behavior indicates that $\mathrm{Cs}$ atoms are loading into the molasses from both the Cs beam from the oven as well as from background vapor. The addition of a Zeeman slowing beam should increase the loading rate while maintaining a low background pressure. Such issues are important for the design of PARCS in which low source-region pressure is desirable to minimize collisional loss of atoms with slow launch velocities.

\section{CONCLUSION}

We anticipate that improvements in the launch and post-cool process will result in colder atom samples $(~ \lesssim 2 \mu \mathrm{K})$. Given such temperatures and our current atom numbers, we already meet the flux requirements to reach our target stability at $\sim 10 \mathrm{~s}$ Ramsey times. We are continuing to investigate source operating parameters and anticipate still larger atom flux numbers can be readily achieved.

\section{ACKNOWLEDGMENT}

The authors would like to thank A. Curtis, D. Lee, D. Smith, and D. Sullivan for helpful suggestions on this manuscript.

\section{REFERENCES}

[1] S. R. Jefferts, D. M. Meekhof, J. H. Shirley, M. Stepanovic, and T. E. Parker, "Accuracy results from NIST-F1 laser-cooled cesium primary frequency standard," in Proc. 2000 IEEE/EIA Int. Freq. Contr. Symp., Kansas City, MO, 2000, pp. 714-717.

[2] E. Simon, P. Laurent, and A. Clairon, "Measurement of the stark shift of the Cs hyperfine splitting in an atomic fountain," Phys. Rev. A, vol. 57, pp. 436-439, 1998.

[3] S. R. Jefferts, T. P. Heavner, L. W. Hollberg, J. Kitching, D. M. Meekhof, T. E. Parker, W. Phillips, S. Rolston, H. G. Robinson, J. H. Shirley, D. B. Sullivan, F. L. Walls, N. Ashby, W. M. Klipstein, L. Maleki, D. Seidel, R. Thompson, S. Wu, L. Young, R. F. C. Vessot, and A. De Marchi, "PARCS: A Primary Atomic Reference Clock in Space," in Proc. 1999 Joint Meet. Eur. Freq. Time Forum and The IEEE Int. Freq. Contr. Symp., Besançon, France, Apr. 1999, pp. 141-144.

[4] P. Laurent, P. Lemonde, E. Simon, G. Santarelli, A. Clairon, N. Dimarcq, P. Petit, C. Audoin, and C. Salomon, "A cold atom clock in the absence of gravity,” Eur. Phys. J. D., vol. 3, pp. 201-204, 1998.

[5] C. Fertig and K. Gibble, "RACE: Laser-cooled Rb microgravity clock," in Proc. 2000 IEEE/EIA Int. Freq. Contr. Symp., Kansas City, MO, June 2000, pp. 676-679.

[6] C. Soloman, J. Dalibard, W. Phillips, A. Clairon, and S. Guellati, "Laser cooling of cesium atoms below $3 \mu \mathrm{K}$," Europhys. Lett., vol. 12, pp. 683-688, 1990. 